\title{
Quality assessment of outcome reporting, publication characteristics and overall methodological quality in trials on synthetic mesh procedures for the treatment of pelvic organ prolapse for development of core outcome sets
}

\author{
Thais Regina de Mattos Lourenço ${ }^{1}$ (D) $\cdot$ Vasilis Pergialiotis ${ }^{2} \cdot$ Constantin M. Durnea $^{3} \cdot$ Abdullatif Elfituri $^{4}$. \\ Jorge Milhem Haddad ${ }^{1}$. Cornelia Betschart ${ }^{5} \cdot$ Gabriele Falconi $^{6} \cdot$ Christiana Campani Nygaard $^{4,7}$. \\ Stergios K. Doumouchtsis ${ }^{2,4,8,9}$. CHORUS: An International Collaboration for Harmonising Outcomes, Research and \\ Standards in Urogynaecology and Women's Health
}

Received: 11 October 2020 / Accepted: 13 January 2021 / Published online: 8 April 2021

(C) The Author(s) 2021

\begin{abstract}
Introduction and hypothesis Variations in outcome measures and reporting of outcomes in trials on surgery for pelvic organ prolapse (POP) using synthetic mesh have been evaluated and reported. However, the quality of outcome reporting, methodology of trials and their publication parameters are important considerations in the process of development of Core Outcome Sets. We aimed to evaluate these characteristics in randomized controlled trials on surgery for POP using mesh.

Methods Secondary analysis of randomized controlled trials on surgical treatments using synthetic mesh for POP previously included in a systematic review developing an inventory of reported outcomes and outcome measures. The methodological quality was investigated with the modified Jadad criteria. Outcome reporting quality was evaluated with the MOMENT criteria. Publication parameters included publishing journal, impact factor and year of publication.

Results Of the 71 previously reviewed studies published from 2000 to 2017, the mean JADAD score was 3.59 and the mean MOMENT score was 4.63. Quality of outcomes $($ MOMENT) was related to methodological quality $(\mathrm{JADAD})($ rho $=0.662 ; p=$ 0.000 ) and to year of publication (rho $=0.262 ; p=0.028$ ).

Conclusions Methodological quality and outcome reporting quality appear correlated. However, publication characteristics do not have strong associations with the methodological quality of the studies. Evaluation of the quality of outcomes, methodology and publication characteristics are all an indispensable part of a staged process for the development of Core Outcome and Outcome Measure Sets.
\end{abstract}

Keywords Pelvic organ prolapse $\cdot$ Mesh prolapse surgery $\cdot$ Core outcome sets $\cdot$ Synthetic mesh

Stergios K. Doumouchtsis

sdoumouc@sgul.ac.uk; sdoum@yahoo.com

1 Department of Obstetrics and Gynaecology, Urogynaecology Division, Hospital das Clínicas da Faculdade de Medicina da Universidade de São Paulo, São Paulo, SP, Brazil

2 Laboratory of Experimental Surgery and Surgical Research N S Christeas, Athens University Medical School, Athens, Greece

3 Northwick Park Hospital, London North West University Healthcare NHS Trust, London, UK

4 Department of Obstetrics and Gynaecology, Epsom \& St Helier University Hospitals NHS Trust, London, UK
5 Department of Gynecology, University Hospital of Zurich, Zurich, Switzerland

6 Department of Obstetrics and Gynaecology, San Bortolo Hospital, Vicenza, Italy

7 Department of Obstetrics and Gynaecology, Hospital São Lucas, Pontifícia Universidade Católica do Rio Grande do Sul, Porto Alegre, Brazil

8 St George's University of London, London, UK

9 American University of the Caribbean, School of Medicine, Pembroke Pines, Florida, USA 


\section{Introduction}

The use of synthetic mesh for pelvic organ prolapse (POP) surgical treatment has recently decreased because of concerns around patient safety. These concerns have been the subject of extensive debates [1], and synthetic meshes for transvaginal POP repair were reclassified by the FDA as high-risk devices [1-4]. A high level of evidence on efficacy and safety through systematic reviews and meta-analyses is warranted to aid clinicians, policy makers and women in choices of treatment for pelvic organ prolapse. However, published data are frequently conflicting possibly on account of arbitrary outcome selection and reporting. Current research evidence is of variable quality and methodology and hence robust practice recommendations are lacking because of limitations in research evidence. Studies have reported on variable outcomes, and comparisons among several trials are not possible because of this heterogeneity [5].

The process of the development of a Core Outcome Set (COS) includes in-depth evaluation of the selection of outcomes reported in primary trials. Trials often use variable methods, and selection of outcomes varies because of authors' expertise, research priorities and objectives, ethical issues and other factors. Frequently, trials on the same treatment intervention have been designed with different methods and study objectives and have been published in various journals (subspecialized, specialized, general journals) over the years. Our previous systematic review developed the inventory of reported outcomes and outcomes measures as a first step in the process of developing relevant COS based on established standards and following well-defined study protocols and high-quality methods [6]. Our systematic reviews on outcome reporting in trials evaluating surgical treatments in different prolapse procedures, incontinence and childbirth trauma [5, 7-12] included assessments of associations between outcome reporting quality, methodological quality and publication characteristics.

Evaluation of the quality outcome reporting and its associations with methodological quality might provide useful evidence in developing a core outcome set for this area of research and possibly provide valuable guidance and directions for future studies.

The aim of this study was to evaluate methodological quality and outcome quality in trials on surgical treatment of POP using synthetic mesh and assess the associations of methodological quality and outcome quality with publication characteristics of the trials including year of publication and journal impact factor.

\section{Materials and methods}

This review is part of CHORUS (An International Collaboration for Harmonizing Outcomes, Research and Standards in
Urogynecology and Women's Health, i-chorus.org). This study was a secondary analysis of data that were part of a recently published systematic review [5]. Ethical approval was not required for this study, as this is a secondary analysis of data included in a previously published systematic review.

The search strategy was described in the original study in accordance with PRISMA guidelines. We used the following keywords and MeSH terms: management, repair, operation and pelvic organ prolapse in the Cochrane, EMBASE, Medline and Scopus databases from inception until September 2017. Randomized controlled trials (RCTs) using synthetic meshes for any type of pelvic prolapse were included (Fig. 1). We excluded retrospective studies, literature reviews, case reports and non-randomized studies. The studies selected are listed in Appendix 1.

The methodological quality of the included trials was evaluated using the modified Jadad score. This is a 5-point scale that evaluates randomization, adequate method for randomization; blinded trial described; adequate method for blinding and if the trial accounts for the patients selected [13]. The outcome quality was assessed using the MOMENT criteria (Management of otitis media with effusion in cleft palate score system), in a 6-point scale. Areas included in the scoring system are stating a primary outcome; explaining if the primary outcome is defined for reproducible measures; stating a secondary outcome; reporting if the secondary outcome is defined as for reproducible measures; explaining if the choice of outcome and if the methods used are designed to improve appropriately the quality of measures [14]. High-quality studies were considered those that reached score $\geq 4$ based on these criteria. All assessments were undertaken independently by two assessors in line with our previous studies and similar research [7-12]. In cases of disagreement, a third assessor reviewed the scores and provided additional scores. The final scores were calculated and reviewed by the senior investigator.

Year and journal of publication and journal impact factor (IF) were retrieved and documented according to Thomson Reuters' (NY, USA) citation reports for obstetrics and gynecology. Statistical analysis was undertaken using SPSS statistical software (IBM Corp., USA). Association among methodological quality, outcome quality, year of publication and journal's impact factor was calculated by non-parametric correlation (Spearman's correlation). Statistical significance was defined as $p<0.05$.

\section{Results}

We reviewed and assessed the 71 RCTs previously included in our systematic review.

Methodological quality, outcome quality and publication parameters are presented in Table 1. Year of publication 
Fig. 1 Randomized controlled trials (RCTs) using synthetic meshes for any type of pelvic prolapse were included

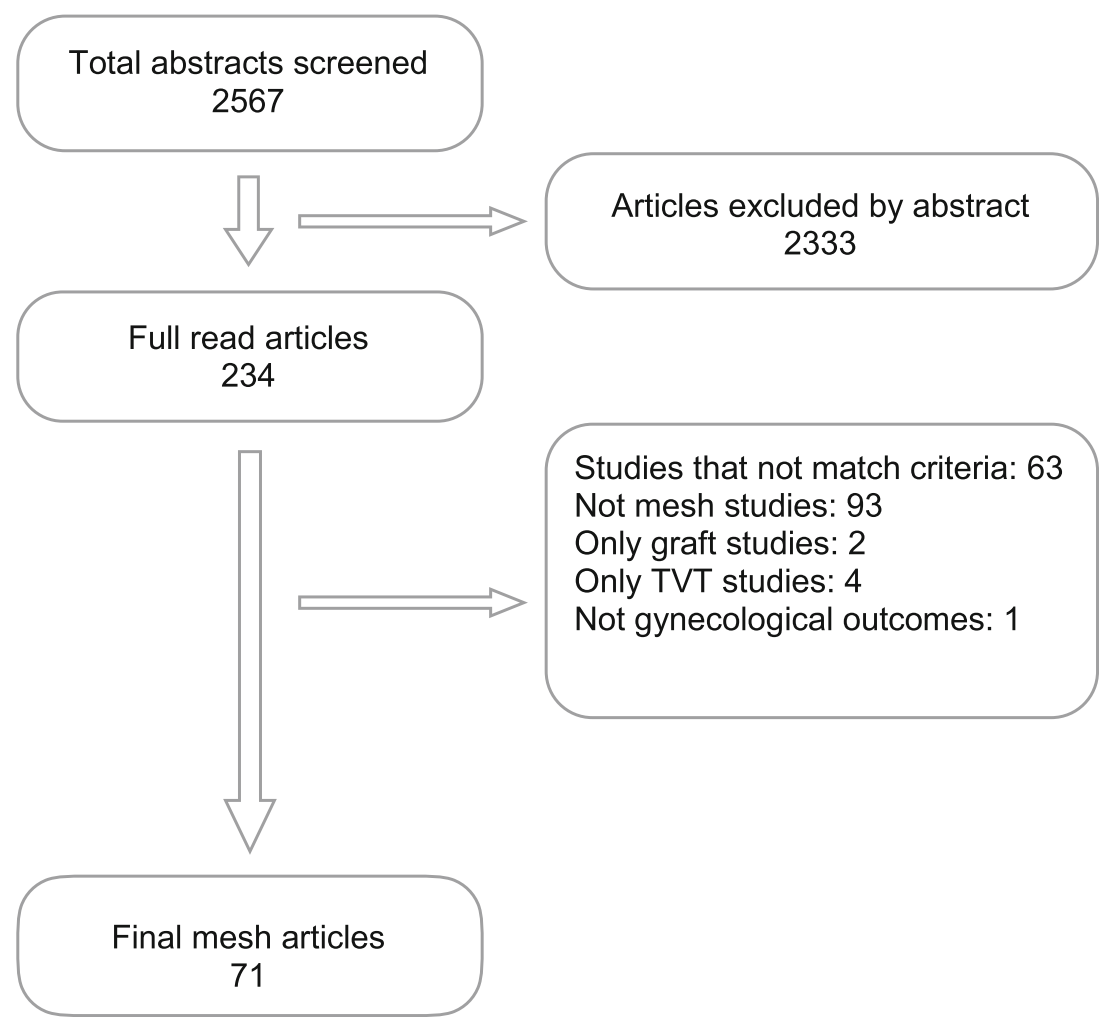

ranged from 2000 to 2017 and follow-up interval ranged from 1.5 to 74 months. The mean Jadad and MOMENT scores were 3.59 and 4.63. Figure 2 shows the quality of outcome (MOMENT) distribution for the total score, and Fig. 3 shows the number of studies scored according to each statement. Fifty-nine studies (83\%) were classified as high-quality outcome reporting, presenting score 4,5 or 6 .

Primary and secondary outcomes were clearly stated in 69 $(97 \%)$ and $46(64 \%)$ of the studies. Only 34 studies $(47 \%)$ provided a rationale for the choice of outcomes but all of them showed methods to improve the outcome measurement described.

A non-parametric correlation revealed that the outcome reporting quality was positively correlated to the methodological quality of the trial $(\mathrm{rho}=0.662 ; p=0.000)$ and to the year of publication ( $r h o=0.262 ; p=0.028$ ). Methodological quality does not appear to be influenced by year of publication (rho $=0.092 ; p=0.444)$ or the journal's impact factor $($ rho $=0.100$; $p=0.417$ ) (Table 2).

\section{Discussion}

In our study, outcome reporting quality ( MOMENT criteria) demonstrated a positive correlation to methodological quality (Jadad score) and to year of publication. The quality of outcome reporting has improved in more recently published trials. However, methodological quality did not have an association with year of publication or the journal's impact factor.

Not surprisingly, methodological quality and outcome reporting quality appear correlated possibly because of the overall study design process and the overall quality of a research protocol. However, such "grading" of the overall quality of a study does not necessarily translate into a publication in a journal with a higher impact factor. This observation highlights the need for harmonization of the quality of methodology and of the reported outcomes and possibly the development of set criteria in research protocols that may assist journal editors in the peer review publication process.

The methodological quality and quality of reported outcomes as well as publication characteristics should be taken into consideration during the process of development of core outcome sets.

One of the strengths of our systematic review is to be the first one to our knowledge evaluating the methodological quality as well as outcome reporting quality in trials using mesh for the treatment of pelvic organ prolapse. We followed a well-established methodology in a standardized manner in order to provide unbiased and objective evaluation of the above-mentioned parameters of the published trials. Another strength of our study was the independent assessments that were undertaken and the process of review and consensus around the final scores of the different domains and items.

However, a number of limitations warrant caution in the interpretation of our results. 
Table 1 Quality of studies

\begin{tabular}{|c|c|c|c|c|c|}
\hline Author & Year & Journal & $\mathrm{IF}^{\mathrm{a}}$ & Jadad & MOMENT \\
\hline Altman et al. & 2011 & New England Journal of Medicine & 29.1 & 4 & 5 \\
\hline Anger et al. & 2014 & Obstetrics and Gynecology & 4.76 & 2 & 5 \\
\hline Barber et al. & 2009 & Obstetrics and Gynecology & 4.69 & 3 & 4 \\
\hline Bradley et al. & 2007 & American Journal of Obstetrics and Gynecology & 4.45 & 3 & 4 \\
\hline Bradley et al. & 2008 & American Journal of Obstetrics and Gynecology & 4.45 & 3 & 4 \\
\hline Carey et al. & 2009 & BJOG: An International Journal of Obstetrics and Gynecology & 4.64 & 3 & 5 \\
\hline Chmielewski et al. & 2011 & American Journal of Obstetrics and Gynecology & 5.34 & 4 & 4 \\
\hline Choe et al. & 2000 & Journal of Urology & 2.64 & 2 & 3 \\
\hline Constantini et al. & 2016 & Journal of Urology & 4.68 & 5 & 6 \\
\hline Constantini et al. & 2007 & European Urology & 5.96 & 3 & 3 \\
\hline Coolen et al. & 2017 & International Urogynecology Journal & 2.078 & 3 & 6 \\
\hline Culligan et al. & 2005 & Obstetrics and Gynecology & 4 & 5 & 6 \\
\hline Culligan et al. & 2013 & Obstetrics and Gynecology & 4.78 & 5 & 6 \\
\hline Cundiff et al. & 2008 & American Journal of Obstetrics and Gynecology & 4.98 & 3 & 4 \\
\hline de Tayrac et al. & 2013 & International Urogynecology Journal & 2.53 & 3 & 5 \\
\hline Delroy et al. & 2013 & International Urogynecology Journal & 2.45 & 5 & 6 \\
\hline Dias et al. & 2016 & Neurourology and Urodynamics & 2.48 & 5 & 6 \\
\hline Ek et al. & 2013 & International Urogynecology Journal & 2.53 & 2 & 4 \\
\hline Ek et al. & 2010 & Neurourology and Urodynamics & 3.01 & 5 & 4 \\
\hline El-Nazer et al. & 2012 & American Journal of Obstetrics and Gynecology & 1.56 & 5 & 5 \\
\hline Farthmann et al. & 2013 & International Urogynecology Journal & 2.45 & 3 & 3 \\
\hline Freeman et al. & 2013 & International Urogynecology Journal & 2.53 & 5 & 6 \\
\hline Glazener et al. & 2016 & Trials & N/A & 4 & 6 \\
\hline Glazener et al. & 2017 & Health Technology Assessment & N/A & 4 & 6 \\
\hline Gupta et al. & 2014 & South African Journal of Obstetrics \& Gynecology & 0.23 & 3 & 4 \\
\hline Halaska et al. & 2012 & American Journal of Obstetrics and Gynecology & 5.32 & 3 & 5 \\
\hline Heinonen et al. & 2011 & European Journal of Obstetrics \& Gynecology and Reproductive Biology & 2.58 & 3 & 5 \\
\hline Hiltunen et al. & 2007 & Obstetrics and Gynecology & 4.45 & 3 & 4 \\
\hline Iglesia et al. & 2010 & Obstetrics and Gynecology & 4.98 & 5 & 6 \\
\hline Lakeman et al. & 2011 & Journal of Sexual Medicine & 3.67 & 3 & 4 \\
\hline Lamblin et al. & 2014 & International Urogynecology Journal & 2.45 & 3 & 5 \\
\hline Lopes et al. & 2010 & International Urogynecology Journal & 2.66 & 3 & 3 \\
\hline Madhuvrata et al. & 2011 & Journal of Obstetrics and Gynecology & 0.75 & 5 & 5 \\
\hline Maher et al. & 2003 & American Journal of Obstetrics and Gynecology & 3.59 & 3 & 5 \\
\hline Maher et al. & 2012 & American Journal of Obstetrics and Gynecology & 5.32 & 5 & 6 \\
\hline Maher et al. & 2011 & American Journal of Obstetrics and Gynecology & 5.34 & 5 & 6 \\
\hline Menefee et al. & 2011 & Obstetrics and Gynecology & 5.34 & 5 & 6 \\
\hline Milani et al. & 2011 & Journal of Sexual Medicine & 3.67 & 3 & 6 \\
\hline Natale et al. & 2009 & International Urogynecology Journal & 2.84 & 3 & 5 \\
\hline Nieminen et al. & 2010 & American Journal of Obstetrics and Gynecology & 4.98 & 3 & 4 \\
\hline Nieminen et al. & 2008 & International Urogynecology Journal & 2.51 & 3 & 2 \\
\hline Noé et al. & 2013 & Archives of Gynecology and Obstetrics & 1.63 & 3 & 2 \\
\hline Noé et al. & 2015 & Journal of Endourology & 2.09 & 3 & 4 \\
\hline Nygaard et al. & 2008 & American Journal of Obstetrics and Gynecology & 4.7 & 2 & 4 \\
\hline Nygaard et al. & 2013 & JAMA - Journal of the American Medical Association & 13.59 & 3 & 4 \\
\hline Paraiso et al. & 2011 & Obstetrics and Gynecology & 5.34 & 5 & 6 \\
\hline Park et al. & 2013 & International Urogynecology Journal & 2.45 & 3 & 5 \\
\hline Qatawneh et al. & 2013 & Gynecological Surgery & 0.46 & 3 & 5 \\
\hline Rahmanou et al. & 2015 & International Urogynecology Journal & 1.83 & 3 & 5 \\
\hline
\end{tabular}


Table 1 (continued)

\begin{tabular}{|c|c|c|c|c|c|}
\hline Author & Year & Journal & $\mathrm{IF}^{\mathrm{a}}$ & Jadad & MOMENT \\
\hline Rane et al. & 2004 & Australian and New Zealand Journal of Obstetrics and Gynecology & 0.87 & 5 & 5 \\
\hline Rondini et al. & 2015 & International Urogynecology Journal & 2.17 & 3 & 4 \\
\hline Roovers et al. & 2005 & Neurourology and Urodynamics & 3.23 & 3 & 5 \\
\hline Roovers et al. & 2004 & BJOG: An International Journal of Obstetrics and Gynecology & 3.01 & 3 & 5 \\
\hline Rudnicki et al. & 2015 & BJOG: An International Journal of Obstetrics and Gynecology & 2.9 & 3 & 3 \\
\hline Rudnicki et al. & 2014 & BJOG: An International Journal of Obstetrics and Gynecology & 2.9 & 3 & 5 \\
\hline Sand et al. & 2001 & American Journal of Obstetrics and Gynecology & 2.72 & 3 & 4 \\
\hline Silveira et al. & 2014 & International Urogynecology Journal & 2.17 & 3 & 5 \\
\hline Shi et al. & 2017 & Medical Science Monitor & N/A & 2 & 3 \\
\hline Sivaslioglu et al. & 2008 & International Urogynecology Journal & 2.79 & 3 & 2 \\
\hline Svabik et al. & 2014 & Ultrasound in Obstetrics and Gynecology & 4.5 & 4 & 5 \\
\hline Tamanini et al. & 2015 & Journal of Urology & 4.68 & 4 & 5 \\
\hline Tamanini et al. & 2013 & International Braz J Urol: official journal of the Brazilian Society of Urology & 1.24 & 4 & 5 \\
\hline Tamanini et al. & 2013 & International Braz J Urol: official journal of the Brazilian Society of Urology & 1.24 & 4 & 5 \\
\hline Tan-Kim et al. & 2014 & International Urogynecology Journal & 2.17 & 5 & 6 \\
\hline Turgal et al. & 2013 & European Journal of Obstetrics \& Gynecology and Reproductive Biology & 2.4 & 3 & 2 \\
\hline Visco et al. & 2008 & International Urogynecology Journal and Pelvic Floor Dysfunct. & 2.51 & 5 & 4 \\
\hline Vollebregt et al. & 2012 & Journal of Sexual Medicine & 3.67 & 5 & 6 \\
\hline Vollebregt et al. & 2011 & BJOG: An International Journal of Obstetrics and Gynecology & 2.96 & 5 & 6 \\
\hline Weber et al. & 2001 & American Journal of Obstetrics and Gynecology & 2.72 & 2 & 3 \\
\hline Withagen et al. & 2011 & Obstetrics and Gynecology & 5.34 & 5 & 6 \\
\hline Yuk et al. & 2012 & Journal of Minimally Invasive Gynecology & 2.1 & 3 & 3 \\
\hline
\end{tabular}

a Journal's impact factor

We evaluated a highly selective cohort of studies leaving out studies that were non-randomized and with different methodologies. Nevertheless, inclusion of a wider variety of studies would be likely to demonstrate wider variations and accentuate our findings. Furthermore, our findings are based on the instruments used and their inherent limitations. Some studies with higher quality protocols but with suboptimal descriptions of these protocol in the published text may have received lower scores than deserved.

Finally, on reviewing the correlation of the quality of the trial and the journal impact factor, we should take into consideration that the choice of the journal a study was published in
Fig. 2 Distribution of scores on quality of outcome reporting (MOMENT scores)

\section{Quality of outcomes (MOMENT score)}

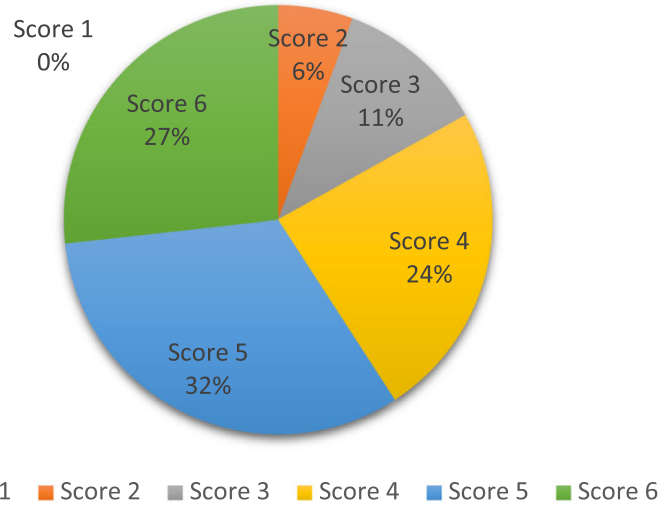


Fig. 3 Number of studies satisfying the specific statements (yes or no) on quality of outcomes' assessment

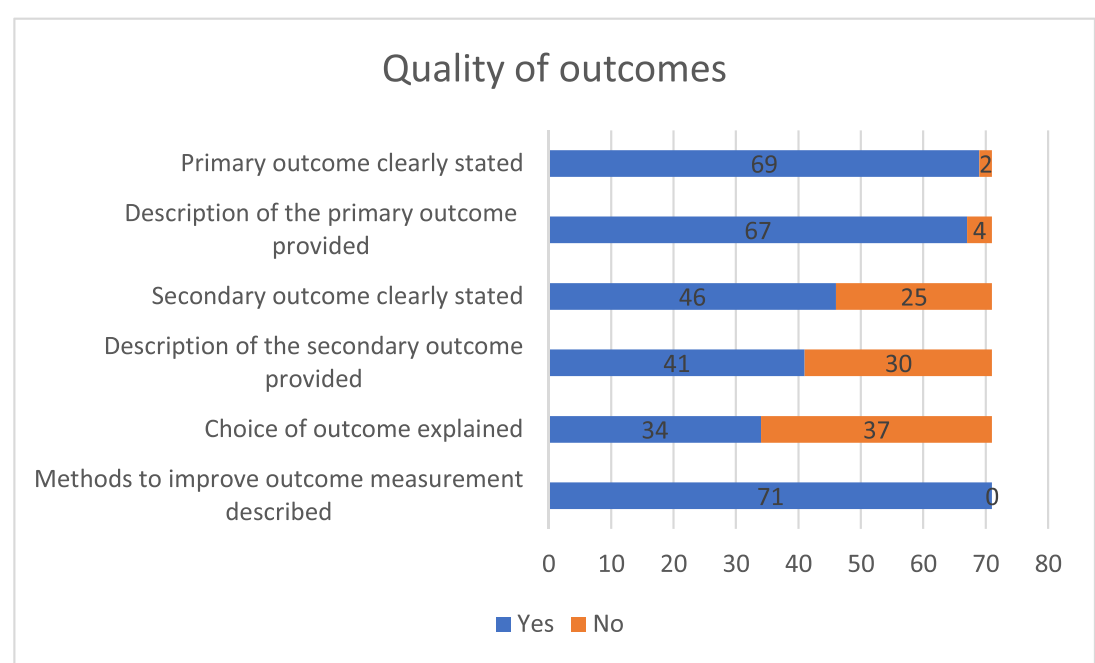

Table 2 - Univariate correlation on publication characteristics, JADAD score and MOMENT score

\begin{tabular}{|c|c|c|c|c|c|c|c|c|c|}
\hline & \multicolumn{3}{|l|}{ MOMENT score } & \multicolumn{3}{|l|}{ JADAD } & \multicolumn{3}{|l|}{$\mathrm{IF}^{\mathrm{b}}$} \\
\hline & Spearman's Rho & $p$ & $N$ & Spearman's Rho & $p$ & $N$ & Spearman's Rho & $p$ & $N$ \\
\hline Year $^{\mathrm{a}}$ & 0,262 & 0,028 & 71 & 0,920 & 0,444 & 71 & $-0,320$ & 0,008 & 68 \\
\hline $\mathrm{IF}^{\mathrm{b}}$ & 0,162 & 0,187 & 68 & 0,100 & 0,417 & 68 & - & - & - \\
\hline JADAD & 0,662 & 0,000 & 71 & - & - & - & - & - & - \\
\hline
\end{tabular}

${ }^{\mathrm{a}}$ Year of publication

b Journal's impact factor

$N$ number

can be influenced by many factors not necessarily associated directly with the quality of the study. For example, a presentation of a study at a conference may be rewarded by an offer of a fast track review of the paper in the journal associated to the society organizing the conference. Some authors may also select a journal to submit based on personal preference, influenced by factors such as journal loyalty of the author, previous or current association with the journal's editorial board or other factors. Hence, this correlation between quality of a trial and journal of publication should always be interpreted with caution for such biases that cannot be weighed.

In the studies included in this evaluation, methodological quality of trials was positively associated with outcome quality but was not strongly associated to year of publication and the journal's impact factor. The instruments and methodology described, already widely used in many fields of medical research, in gynecology, obstetrics and urogynecology, were applied to analyze in a standardized way important parameters of published research [5]. This assessment is in our opinion a fundamental prerequisite in the process of developing a high quality $\operatorname{COS}$ in line with the standards established by the COMET, COSMIN and CROWN initiatives.
Nevertheless, we believe that this study may also provide invaluable guidance for improving better selection of outcomes and measurement tools, outcome reporting, research methods and publication strategies for future research in this area. Harmonized methodology and outcome selection and reporting may improve the comparability of primary research, which in turn may inform robust meta-analyses and eventually improve clinical practice.

\section{(C)) CHORUS}

Supplementary Information The online version contains supplementary material available at https://doi.org/10.1007/s00192-021-04749-3.

Acknowledgements CHORUS: An International Collaboration for Harmonising Outcomes, Research and Standards in Urogynaecology and Women's Health (https://i-chorus.org/). 
Author's participation Lourenço TRM: data analysis, manuscript writing.

Pergialiotis V: data analysis, manuscript editing.

Durnea CM: data collection, data analysis.

Elfituri A: data collection, data analysis.

Haddad JM: manuscript writing/editing.

Betschart C: manuscript writing/editing.

Falconi G: manuscript writing/editing.

Nygaard CC: manuscript writing/editing.

Doumouchtsis SK: project development, manuscript writing, data analysis.

\section{Declarations}

Conflicts of interest None.

\section{References}

1. Souders CP, Eilber KS, McClelland L, Wood LN, Soulders AR, Steiner V, et al. The truth behind transvaginal mesh litigation: devices, timelines, and provider characteristics. Female Pelvic Med Reconstr Surg. 2018;24(1):21-5.

2. Chughtai B, Sedrakyan A, Mao J, Eilber KS, Anger JT, Clemens JQ. Is vaginal mesh a stimulus of autoimmune disease? Am J Obstet Gynecol. 2017;216(5):495. e1-495.e7.

3. Karmakar D, Dwyer PL. Failure of expectations in vaginal surgery: lack of appropriate consent, goals and expectations of surgery. Curr Urol Rep. 2016;17(12):87.

4. Federal Register/Vol. 81 No. 2/Tuesday, January 5, 2016/Rules and Regulation FDA 21 CFR Part 884 Avalaible at https://www.gpo. gov/fdsys/pkg/FR-2016-11-22/pdf/FR-2016-11-22.pdf

5. de Mattos Lourenco TR, Pergialiotis V, Duffy JMN, Durnea C, Elfituri A, Haddad JM, et al. A systematic review on reporting outcomes and outcome measures in trials on synthetic mesh procedures for pelvic organ prolapse: urgent action is needed to improve quality of research. Neurourol Urodyn. 2019;38(2):509-24.

6. Kirkham JJ, Davis K, Altman DG, Blazeby JM, Clarke M, Tunis S, et al. Core outcome set-STAndards for development: the COSSTAD recommendations. PLoS Med. 2017;14(11):e1002447.
7. Durnea CM, Pergialiotis V, Duffy JMN, Bergstrom L, Elfituri A, Doumouchtsis S. A systematic review of outcome and outcome measure reporting in randomised trials evaluating surgical interventions for anterior-compartment vaginal prolapse: a call to action to develop a core outcome set. Int Urogynecol J. 2018;29(12):1727-45.

8. de Mattos Lourenço TR, Pergialiotis V, Durnea CM, Elfituri A, Haddad JM, Betschart C, et al. A systematic review of reported outcomes and outcome measures in randomized controlled trials on apical prolapse surgery. Int J Gynecol Obstet. 2019;145(1):4 11.

9. Lourenço TRM, Pergialiotis V, Durnea CM, Alfituri A, Haddad JM, Betschart C, et al. A systematic review of reported outcomes and outcome measures in randomized trials evaluating surgical interventions for posterior vaginal prolapse to aid development of a core outcome set. Int J Gynecol Obstet. 2020;148(3):271-81.

10. Doumouchtsis S, Rada MP, Pergialiotis V, Falconi G, Haddad JM, Betschart C. A protocol for developing, disseminating, and implementing a core outcome set (COS) for childbirth pelvic floor trauma research. BMC Pregnancy Childbirth. 2020;20(1):376.

11. Doumouchtsis SK, Pookarnjanamorakot P, Durnea C, Zini M, Elfituri A, Haddad JM, et al. A systematic review on outcome reporting in randomized controlled trials on surgical interventions for female stress urinary incontinence: a call to develop a core outcome set. BJOG. 2019;126(12):1417-22.

12. Rada MP, Pergialiotis V, Betschart C, Falconi G, Haddad JM, Doumouchtsis SK. A protocol for developing, disseminating, and implementing a core outcome set for stress urinary incontinence. Medicine. 2019;98(37):e16876.

13. Kennelley J. Methodological approach to assessing the evidence. Reducing racial and ethnic disparities in reproductive and perinatal outcomes the evidence from population-based interventions. New York: Springer; 2011. p. 7-20.

14. Harman NL, Bruce IA, Callery P, Tierney S, Sharif MO, O'Brien $\mathrm{K}$, et al. MOMENT (Management of otitis media with effusion in cleft palate): protocol for a systematic review of the literature and identification of a core outcome set using a Delphi survey. Trials. 2013;14:70.

Publisher's note Springer Nature remains neutral with regard to jurisdictional claims in published maps and institutional affiliations. 